\title{
THE INFLUENCE OF VARIATIONS OF GEOMETRICAL PARAMETERS ON THE NOTCHING STRESS INTENSITY FACTORS OF CYLINDRICAL SHELLS
}

\author{
Hassane Moustabchir, Moulay A. Hamdi Alaoui, Abdelhak Babaoui \\ Équipe Science et Ingénierie des Matériaux (ESIM), Département de Physique Faculté des Sciences et Techniques \\ Errachidia, Université My Ismanl, Boutalamine, Errachidia, Morocco \\ Karl D. Dearn, Catalin I. Pruncu \\ School of Mechanical Engineering, University of Birmingham, United Kingdom \\ e-mail: c.i.pruncu@bham.ac.uk
}

Zitoune Azari

LaBPS, Ecole Nationale d'Ingénieurs de Metz, Ile du Saulcy, Metz, France

\begin{abstract}
The modern approach of Virtual Engineering allows one to detect with some accuracy the residual life of components especially free of cracks. The life estimation becomes cumbersome when the components contain a crack. A straightforward formulation requires a parameter that considers geometrical constraints and materials properties. The magnitude of the stress singularity developed by the tip of a crack, needs to be expressed by the Stress Intensity Factors (SIF). In order to prove the validity of the results, calibration by experimental and/or analytical technique is required. To have a better understanding of this parameter, in the first part of this paper an analytical model to compute the SIF connected to crack propagation into Mode I has been implemented. The case study displays a pipeline component with a crack defect submitted to internal pressure. Therefore, an appropriate correlation between the analytical approach and numerical simulation has been established embedded.
\end{abstract}

Keywords: crack, Finite Element (FE), internal pressure, Stress Intensity Factors (SIF)

\section{Introduction}

Mechanical engineering, manufacturing and/or materials performance are still attractive fields of research because of the existence of imperfections in the material components. This open question is notable on pipeline structures that seem to impose an effective challenge due to cumulative price between residual life estimation and capital costs (Schoots et al., 2011). The future development of manufacturing capabilities and the industrial applications of steel cylinders, tailored in this field, is recognized to be very sensitive to the defects occurrence.

The sizes of defects deal with the magnitude of industrial accidents in such cylinders (Staat and Duc, 2007). In technological product innovation, the fracture properties of steel cylinders with defects are very important for their engineering design and application. In the final product, defects may arise in different ways because of cumulative loading action (i.e. internal pressure, temperature gradient, friction between soil/pipes interface and so on, see in Fig. 1).

The crack like "cavities" in Buried Pipeline was determined in (Lee and Choi, 1999) using the maximum equivalent stresses. The simplified approach show the effects of cavities on maximum equivalent stresses of the buried pipeline, and the stresses associated of two main factors such as size and location. Here, may be worth to note that there is a link between the effects of location while the cavities are at the same depth. It was observed when the cavities develop larger than 


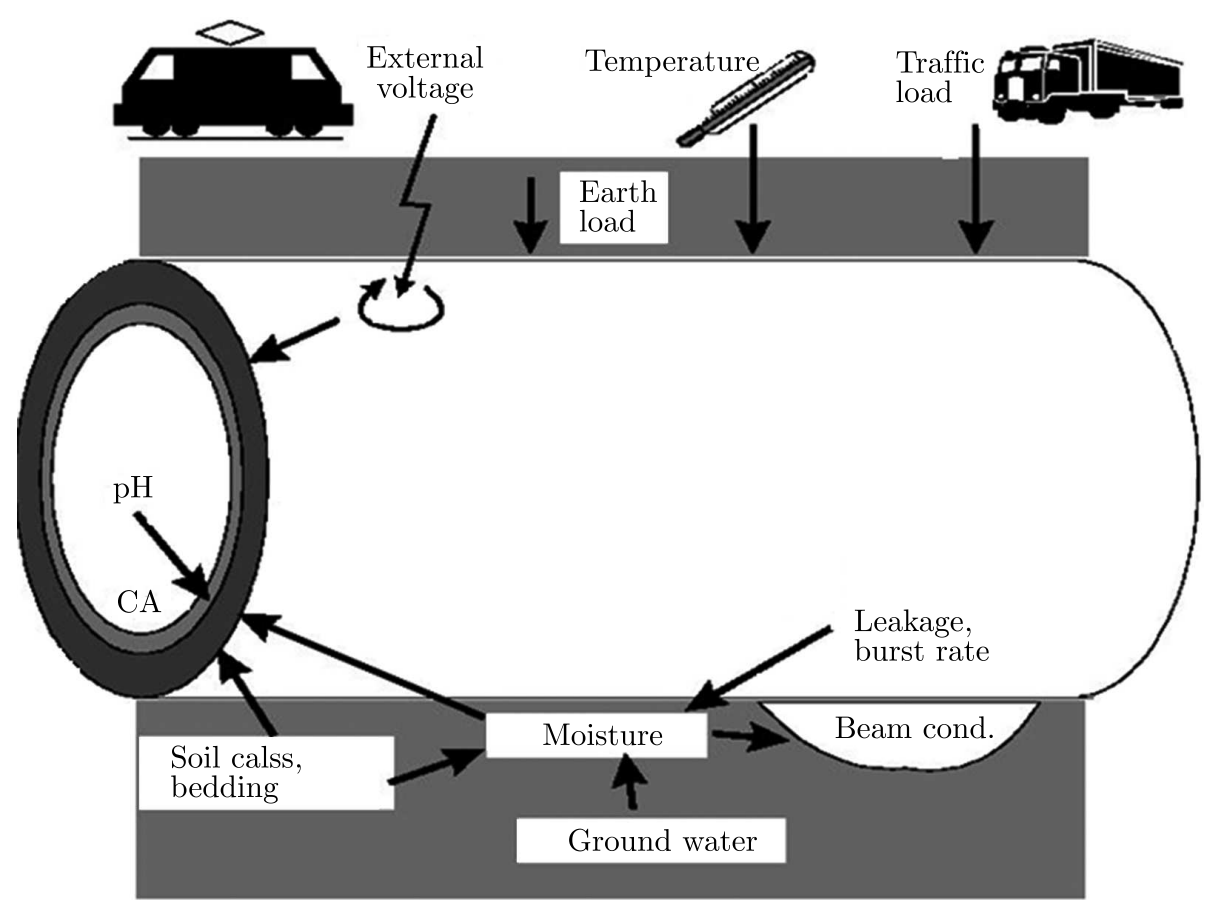

Fig. 1. Example of loads generated in a pipe section

pipeline diameter, the effects of cavities located below the pipeline are found to be larger than those located above the pipeline.

A method to solve the fracture behavior of cracked pipes can be settled using the $J$ - $Q$ approach, by using a plane-strain constrain to characterize the effects of control on cleavage fracture behavior. This method allows good assessment for material with cleavage fracture behavior, because the toughness data appear more applicable for cleavage fracture predictions. The approach works well on pressurized pipelines and cylindrical vessels compared to standard methods with an assumption valid on deep notch fracture specimens under bend loading (Cravero and Ruggieri, 2005).

The impact of geometric parameters as well as the crack-tip opening displacement (CTOD) and the crack tip opening angle (CTOA) allows accurate solution in the case of a dam aged pipeline. A digital camera and image analysis software may offer evidence of the progression of the crack tip and may estimate accurately the CTOA (Darcis et al., 2008). This consideration became effective for the fracture evaluation in a particular case of ductile behavior. However, this imposes some limitation in a distinct type of engineering situations, namely situations in which ductile fracture can develop quantitatively a priori and occur in mode I, as it is frequently in the case of pipelines and pressure vessels (Corigliano et al., 1999). To predict experimentally the activity of burst pressures in a thin-walled gas pipeline containing longitudinal cracks, a micromechanics approach based upon the computational cell methodology enclosed in the Gurson-Tvergaard model and a deformation-based approach settled on the CTOA criterion may be relevant. The verification methodology shows the potential predictive capability of the cell approach incorporating the GT model, particularly for moderate to deep crack size to pipe thickness ratios $(a / t)$. However, burst pressure predictions for a shallow crack pipe specimen displayed poor agreement with experiments and largely overestimated the measured failure pressures (Rugieri and Dotta, 2011).

By using the constraint $R^{*}$, the effects of geometries and crack dimensions was determined on axially cracked pipes and SENT (Single Edge Notched Tension) specimens (Tan et al., 2015). The creep crack-tip constraint are significant when changes are made to some parameters, for 
examples, by increasing crack depth, crack length linked to $D / t$ (diameter/thickness). A constraint level as a function of test specimens from high to low may be ordered in the following manner: CT (Compact Tension), CST (C-Shaped Tension), SENB (Single-Edge Notched Bend), SENT and CCT (Center-Cracked Tension). It was observed that the constraint developed by the pin-loaded SENT specimen was almost the same as that of the clamped SENT specimen. Besides, the creep constraint $R^{*}$ of SENT specimen increases with increasing crack depth $a / W$ (crack depth/specimen width) and specimen width $W$.

At low temperature $\left(-40^{\circ} \mathrm{C}\right.$ to $\left.-60^{\circ} \mathrm{C}\right)$, crack extension vs. time is a critical importance situation because the dynamic crack propagation is very often encountered. To determine fitness for service of arctic pipelines, bursting tests of line pipes samples at low temperature were carried out within a reasonable uncertainty by using timing wires. The determination highlights the axial crack fracture mechanism in a quasi-cleavage manner in the center of the pipe wall showing width of the shear lips be positively correlated with test temperature and burst pressure (Murtagian et al., 2005). Recently, Nordhagen et al. (2014) proposed a numerical solution using a simple artificial fluid-structure interaction model validated using an advanced ideal fluidstructure interaction proposal. It appears that average crack speed is most sensitive to pipe thickness, followed by initial pressure, Cockcroft-Latham fracture parameter, decay length, yield strength, pipe diameter and work-hardening.

In order to ensure safe design of pipelines, new methods are compulsory; they can be based on depth knowledge and appropriate characterization of material resistance. However, it is necessary to find a combination between quasi-static test and dynamic drop weight tear testing (DWTT) on modified specimens with pre-fatigued crack where the application of the cohesive zone (CZ) and Gurson-Tveergard-Needleman (GTN) models to describe ductile material damage may be applied. This approach contributes to an increase in the understanding of major parameters controlling ductile fracture propagation and helping to establish a reliable procedure for safe design of new high-capacity pipelines with regard to crack arrest (Scheider et al., 2014).

In this paper, steel cylinders submitted to internal pressure with longitudinal surface defects are evaluated. This kind of longitudinal defects develop, usually, on the external part of the pipe surface in the axial way. The major consideration is dedicated to calculating contributions of the SIF in the steel cylinders submitted to different levels of loading.

To be noted, this survey attempts to confront also the solutions proposed in literature (Heliot et al., 1997; McGowan and Raymund, 1997; Shin and Cai, 2004; You et al., 2007) devoted to calculation of SIFs of pressurized cylinders with longitudinal cracks. In fact, this paper permits one to consolidate a robust strategy, suitable for designing industrial components by using the Finite Element Methods (FEM) confidently. So, using the analytical models cited in the literature allows eliminating "the industrial fear" that appears while an industrial user comes in the contact with "the black box" approach of the FEM tools.

In Fig. 2, we present respectively a physical problem where the SIFs can be applied successfully along the crack front, the component from a steel cylinder with different external surface crack sizes. The internal pressure enforced to fracture the wall size was $P=1.5 \mathrm{MPa}$ that corresponded to a working pressure of a pipeline platform. In the present configuration (i.e. sketched in Fig. 2a and 2b), it is assumed that both values of the SIF for Mode I, namely, $K_{I}$ parameter reach the largest values at the deepest points of different external surface cracks and, besides, decrease from the deepest point down to the surface point along the crack front. A mathematical formulation of SIFs values along the axial external surface cracks can be very well expressed by a polynomial equation following the point locations along the crack fronts by using six order polynomials

$$
K_{I}=\sum_{i=0}^{6} A_{i}\left(\frac{2 \phi}{\pi}\right)^{i}
$$


where $A_{i}$ are constants that depend on the crack type, crack size, internal pressure, dimensions and of course the material of the cylinder. Details on the conditions of the present formulations (i.e. while the internal pressure is imposed) and all constants for different axial external surface cracks are listed in (Su and Gouri, 1999).

(a)

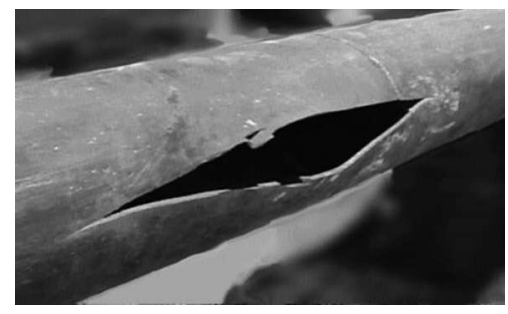

(b)

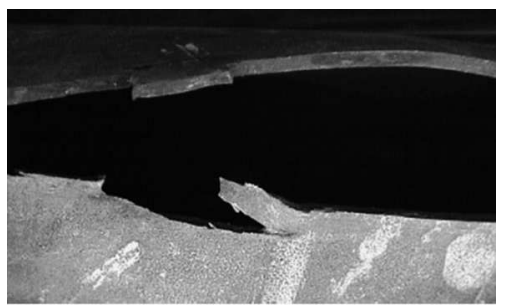

Fig. 2. Photographs of an axial crack in an engineering-structure caused by the applied load (the case of a steel gas cylinder)

\section{Stress intensity factors (SIF) for pipes in service section}

Critical temperature, pressure and the fluid stream can activate together a complex mechanism in the materials behavior while flaw geometries (having different shape) can easily initiate and propagate. To easy solve this challenge, we only consider a hypothetical case of axial flaws at the surface of a pipe (Fig. 3).

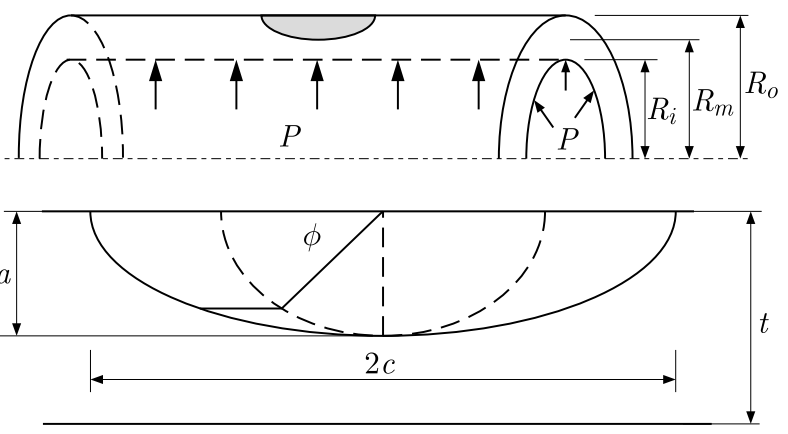

Fig. 3. Pipe geometry containing longitudinal surface flaw

Thus, in the simplified approach connected to the above flow configuration, a symmetry dimension of the structure may require. Once established a convenient methodology to run typical experimental processes, such as this symmetrical geometry, allows detecting accurately the values of $K_{I}$. The computation technique needs to sustain this strategy. There, dynamic loading events such as pressure surges and external impact are ignored, and the expected operating conditions of the buried pipeline are split into static contributions from the internal pressure, loading moment and tension stress.

Since each of these stress components creates mode-I opening of the flaw, individual SIF can be superposed to give a net value of $K_{I}$

$$
K_{I N E T T} \approx K_{I P}+K_{I M}+K_{I T}
$$

with: $K_{I P}$ a factor of internal pressure, $K_{I M}$ a factor of bending load, $K_{I T}$ a factor tension loading.

A minimum assumption to avoid crack propagation, denoted by as well as the material fracture toughness $\left(K_{I C}\right)$, must be sufficient to overcome the combined constraints of other factors. The embedded formulation of the cumulative action of these "external" SIFs allows 
propagation of the cracks, when the critical Stress Intensity Factors SIF's(crit) (i.e. $K_{I C}$ ) become lower with respect to the net value of $K_{I}$

$$
K_{I P}+K_{I M}+K_{I T} \geqslant K_{I C}
$$

So, we notice the primary and most important problem in fracture analysis that the pressure vessel requires determination of the nett value of the SIF of the crack surface. The approach presented in this survey provides a comprehension range of results of SIFs for external surface cracks in a cylindrical fissure vessel.

A pipeline structure submitted to internal loading situations may develop the following state that is displayed in Figs. 4a-4c, where the effect of internal pressure can be examined for three different conditions. In the first case (i.e. Fig. 4a), we may consider only the pressure loading condition. In the second case (i.e. Fig. 4b), the pipe segment is assumed to have 'open ends' (i.e. the axial force at the ends due to pressure is zero). Thirdly, a 'closed end' situation is exhibited by applying the corresponding axial force (end-load) to the ends due to the applied pressure. In reality, the net axial force in a pipeline section may change from tensile to compressive loading. These loading sequences correspond to realistic situations encountered in pipelines. However, differences between the results obtained by the applied loads in a proportional or non-proportional manner could be another problem.

(a)

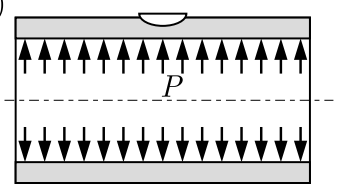

(b)

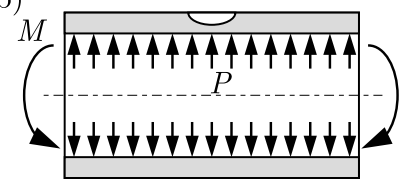

(c)

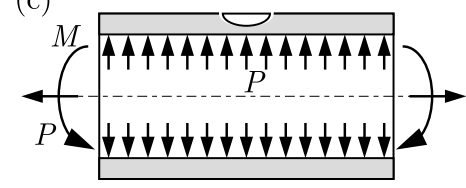

Fig. 4. Different load situations embedded into pipe configuration

When the pipe is loaded under bending routine along with internal pressure, a varying bending moment distribution along the length may be noted. This is caused by nonlinear geometry effects. Hence, in simulations with internal pressure, symmetry boundary conditions at the mid-length of the pipe are imposed through another set of MPC's. This facilitates direct calculation of the resisting moment at the mid-section.

However, has been it verified that the computed fracture parameters are not affected by these MPC's at the mid-section. Numerical analyses (i.e. based on the Finite Element Method (FEM) approach) could be a better tool to simulate the real life of pipe structures (containing flow) and, besides, to corroborate the analytical results with the numerical approximation.

\section{Stress Intensity Factor (SIF) evaluation}

The state of crack growth from a given longitudinal notch in a pipe depends somehow on the nature of the dominant stress state in the component. This reflects the nature of stress intensity ahead of the crack tip. In this specific case of a cracked component, the available evaluation routes for determination of the SIFs have been derived by using both analytical and numerical techniques. The introduction of the $K$ concept (link to the toughness in terms of critical values) proves to be a special value for studies on fracture and/or fatigue cracking (Tada et al., 2000).

A number of researchers have published particular solutions of SIFs for a great range of notch profiles and loading conditions. Raju and Newman (1982) and Anderson (1999), in particular, developed numerical solutions for internally pressurized components containing semi-elliptical shapes with the aspect ratio $\alpha=a / b$ and relative depth $\xi=a / t$ of the deepest point $A$ on the defect front, where $a$ is the maximum crack depth. The fracture analysis of the above cracked 
shell is very useful because some recommendations propose replacing the actual surface defect by an equivalent semi-elliptical crack.

The approach of SIF derived by Raju and Newman (1982) has been taken as the base framework for semi-elliptical cracks. The crack profile has been defined in terms of the shape factor $Q$ approximated by the following expressions

$$
Q= \begin{cases}1+1.464\left(\frac{a}{c}\right)^{1.65} & \text { for } \quad \frac{a}{c}<1 \\ 1+1.464\left(\frac{c}{a}\right)^{1.65} & \text { for } \quad \frac{a}{c}>1\end{cases}
$$

This approximation permits accurate estimation even in a particular case, where the correction factor developed in (ASME, 1981) works as a general model requiring only the first part of equation (3.1). The general hypothesis is

$$
Q=1+1.464\left(\frac{a}{c}\right)^{1.65}
$$

Further, the SIF $K_{I}$ is evaluated in terms of the Lamé average hoop stress as

$$
K_{I P}=\frac{P R_{m}}{t} \sqrt{\frac{\pi a}{Q}} Y\left(\frac{a}{t}, \frac{a}{2 c}, \frac{R_{0}}{t}\right)
$$

where $P$ is the internal pressure, $R_{m}$ and $R_{0}$ are the mean and outer radii of the pipe, respectively, $t$ is pipe thickness, $a$ is depth and $2 c$ is crack length.

The formulation expressed in equation (3.3) covers the range of pipes with $R / t \leqslant 10$, considered in the present survey. It is believed that the approximation is quite good. However, to check the result, an interpolation between the previous solution and the numerical results presented by Stonesifer et al. (1992) for the geometrical correction $Y$ has been made. We noticed that equation (3.3) is valid in the case of pipes with ratios up to $R / t=40$. Finally, for consistent results, the stress intensity factor has been evaluated by the formula presented in (Zahor, 1991) that partially agree with (Raju and Newman, 1982) and with the same limitations on the $R / t$ ratio. The formulation is expressed in the following manner

$$
K_{I P}=P R_{i} \sqrt{\frac{\pi}{t}}\left(0.25+\frac{0.42 \alpha+0.21 \alpha^{2}}{[0.11(R / t)-0.1]^{0.16}}\right)
$$

where the shape factor $\alpha$ is defined

$$
\alpha=\frac{a}{t}\left(\frac{c}{a}\right)^{0.58}
$$

Subsequently, the stress intensity factor has been determined for a through wall crack, adopting two different expressions. The first agrees with (Zahor, 1991), described as

$$
K_{I P}=\frac{P R_{i}}{t} \sqrt{\pi a}\left(1+0.0724 \rho+0.649 \rho^{2}-0.233 \rho^{3}+0.0382 \rho^{4}-0.00235 \rho^{5}\right)
$$

with $\rho=c / \sqrt{R_{i} t}$.

In SAQ Handbook (Anderson, 1999), the Stress Intensity Factor $K$ is defined as

$$
K_{I P}=\sqrt{\pi a} \sum_{i=0}^{3} \sigma_{i} Y_{i}\left(\frac{a}{t}, \frac{a}{2 c}, \frac{R_{m}}{t}\right)
$$

where $\sigma_{i}$ are the components which define the stress distribution though the cylinder wall, according to

$$
\sigma_{u}=\sum_{i=0}^{3} \sigma_{i}\left(\frac{u^{i}}{a}\right)
$$


The co-ordinate $u$ is defined through the remaining wall thickness ( $u$ varies from the crack depth $a$ to the wall thickness $t$ ). The geometrical functions $Y_{i}$ are tabulated in (Raju and Newman, 1982).

This analytical correction has been confronted to the particular solution of (Zahoor, 1991). By using this formulation, the key form expression can be settled following the boundary correction factor $Y$ applied in Eq. (3.3)

$$
Y=1.12+0.053 \xi+\frac{1+0.02 \xi+0.0191 \xi^{2}}{1400}\left(20-\frac{R_{m}}{t}\right)^{2}
$$

with

$$
\xi=\frac{a}{t} \cdot \frac{a}{2 c}
$$

The outcomes derived from equation (3.8) are plotted in Fig. 5.

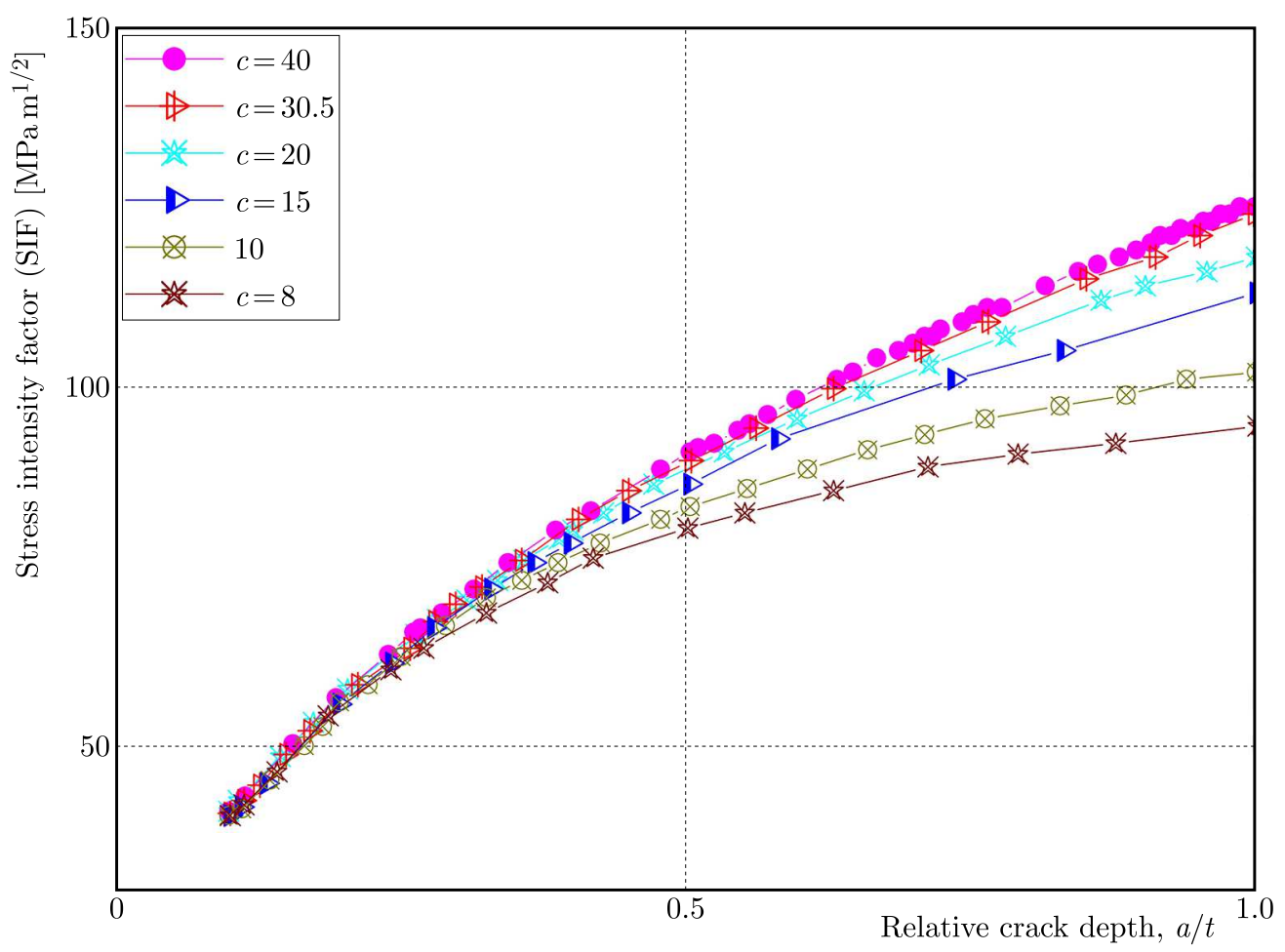

Fig. 5. Variations of $K_{I}$ with relative crack depth determined by Eq. (3.8)

Figure 5 displays the SIFs of steel cylinders with axial surface cracks (considering different lengths of flow), respecting the condition entailed in ( $\mathrm{Su}$ and Bhuyan Gouri, 1999; Anderson, 1994). In a particular state (depending on cylinders with axial cracks), the stress intensity factor can be expressed respecting the following state of different relative crack sizes $2 c / t$

$$
K_{I P}=0.4294\left(\frac{2 c}{t}\right)^{3}-6.1122\left(\frac{2 c}{t}\right)^{2}+49.489 \frac{2 c}{t}-4.1444
$$

This expression is valid only in the range of $2.6<2 c / t<10.4$.

Vis a vis DN100 ABS pipe, in the case when the internal pressure is imposed, the SIF aroused can be calculated using the FE data considering different flaw depths. The accuracy of the results may be enhanced using a polynomial approximation as stated below

$$
K_{I P}=32.149-82.294 \sqrt{a} \frac{a}{t}+263.661 \sqrt{a}\left(\frac{a}{t}\right)^{2}-111.753 \sqrt{a}\left(\frac{a}{t}\right)^{3}
$$


In this configuration, the geometric correction factor have the form

$$
Y=\frac{K_{I P}}{\sigma_{I P} \sqrt{\pi a}}=1.824-4.669 \frac{a}{t}+14.959\left(\frac{a}{t}\right)^{2}-6.341\left(\frac{a}{t}\right)^{3}
$$

where $\sigma_{I P}$ is the membrane hoop stress of the pipe bore surface derived from the Lamé equation for a thick-walled cylinder

$$
\sigma_{I P}=\frac{P\left(R_{i}^{2}+R_{0}^{2}\right.}{R_{0}^{2}-R_{i}^{2}}
$$

where $R_{0}$ and $R_{i}$ are the outer and internal radii of the pipe, respectively.

\section{FE analysis}

The particular model depicted in Fig. 3 highlights a longitudinal crack surface in a pipe under internal pressure $P$ with configuration translated into numerical computation. The labels in the above sketch represent the characteristics of the pipe studied, which matches with a real situation. There, the mean radius and thickness of the pipe are denoted by $R_{m}$ and $t$, respectively. The crack length is characterized by $2 c$ and the geometry is defined such that the depth of the crack is $a$. Four different values of $R_{m} / t$ are employed in the present work and different values of $2 c$ are considered, namely, $2 c=16,20,30,40,61$ and $80 \mathrm{~mm}$. In this configuration, the internal pressure is settled $P=1.5 \mathrm{MPa}$. The $3 \mathrm{D}$ elastic computation approach under FE strategy has been implemented for this case of a longitudinal crack surface. Figure 6 shows details of the configuration. The methodology has been performed using a general-purpose FE program (CASTEM, 2014). The material tensile properties introduced in the FE analysis are assumed to obey the Ramberg-Osgood (R-O) equation

$$
\frac{\varepsilon}{\varepsilon_{0}}=\frac{\sigma}{\sigma_{y}}+\alpha \frac{\sigma}{\sigma_{y}}
$$

where $\varepsilon_{0}, \sigma_{y}$, and $\alpha$ are constants, $E \varepsilon_{0}=\sigma_{y}$, where $E$ is Young's modulus.

In Table 1, mechanical properties of the material evaluated in this paper which is steel P264GH are given.

\section{Table 1}

\begin{tabular}{|l|c|}
\hline \multicolumn{1}{|c|}{ Characteristic } & Values \\
\hline \hline Elastic strain & $\varepsilon_{0}=0.0016$ \\
\hline$\alpha=1.86$ & constant \\
\hline Modulus of elasticity & $E=207000 \mathrm{MPa}$ \\
\hline Poisson's ratio & $\nu=0.3$ \\
\hline Yield strength & $\sigma_{y}=340 \mathrm{MPa}$ \\
\hline Ultimate tensile strength & $R_{m}=440 \mathrm{MPa}$ \\
\hline Elongation to fracture & $A=35 \%$ \\
\hline
\end{tabular}

The shape of the crack embedded in the pipe configuration during simulation is sketched in Fig. 6. In the programing procedure, only a half of the structure has been taken in account to reduce the resources needed for simulation. The structural mesh, having a progressive density of the mesh in front of the crack, has been embedded in order to stimulate the stress singularity. This technique of mesh densities near the notch in the pipeline network was considered with success in the particular case of fracture defect assessment in (Moustabchir et al.. 2015). 

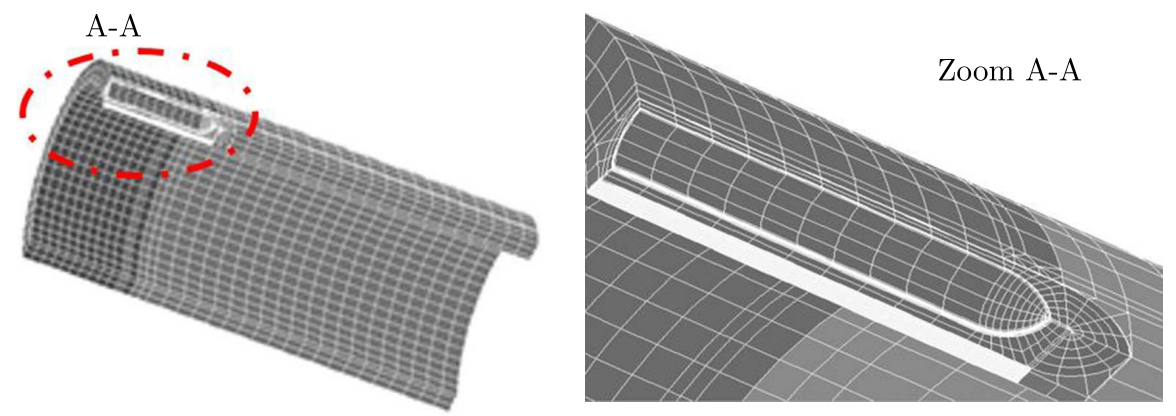

Fig. 6. Finite element configuration of a half of pipe with a longitudinal flaw

\section{Discussions}

In this paper, the engineering $K_{I}$ parameter equations for an axial crack surface located in a pipe under internal pressure are throughly considered. The proposed approach offers details concerning the results obtained using an analytical method and a 3D computation technique. The $K_{I}$ parameter determined obeys R-O materials laws in the case of plastic deformation in a partially and fully plastic situation.

The values of the $K_{I}$ parameter have been determined considering a cylinder containing a defined crack size (i.e. crack surface). A summary regarding the results obtained using these methods of different axial crack surfaces are shown in Figs. 7a and 7b.

Figure 7a presents variations of the SIF values at the deepest points (i.e. crack fronts linked to the wall structure) of the external axial crack surface in the cylinders of different relative crack depths $a / t$ (crack). The results determined here prove a good approximation between the FE outcome and literature determinations.

(a)

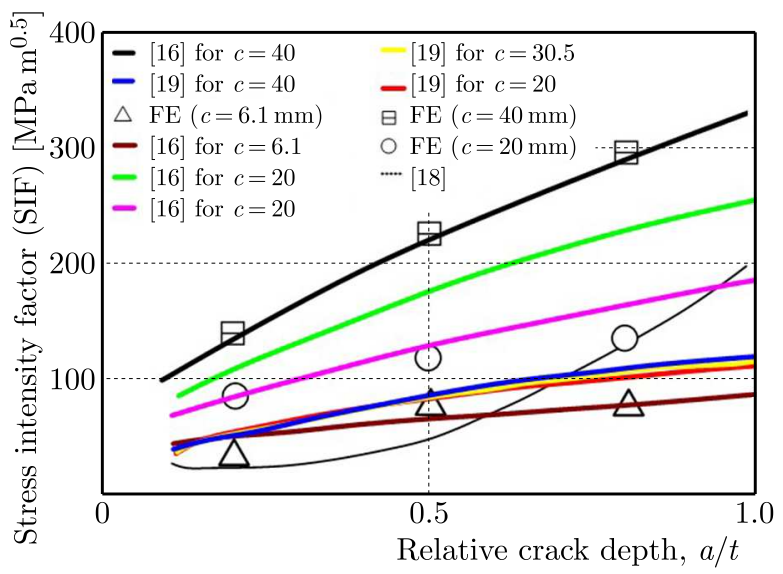

(b)

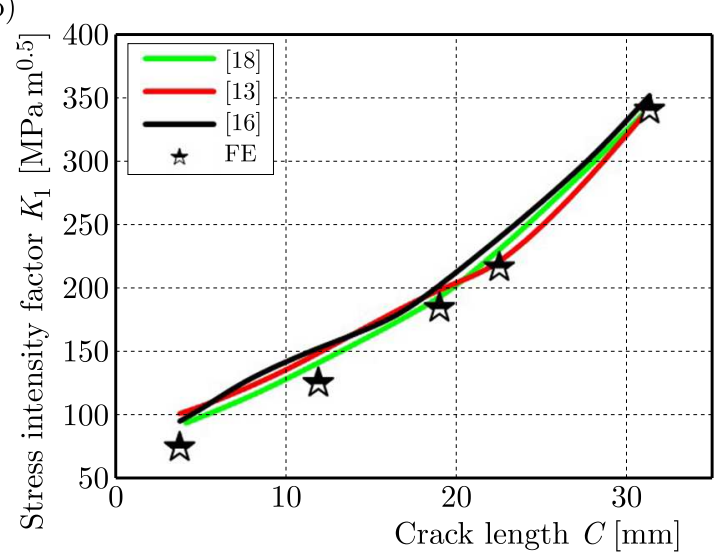

Fig. 7. (a) Variations of the $K_{I}$ parameter at the deepest point for a relative crack depth, (b) variations of $K_{I}$ parameter versus length of the crack; [13] - Nordhagen et al. (2014), [16] - Scheider et al. (2014),

[18] - Schin and Cai (2004), [19] - Staat and Duc (2007)

Figure $7 \mathrm{~b}$ deals with the effect of crack length, determining the evolution of SIF values. These results correspond to a certain situation, where the axial crack surface is characterized by the following dimensions $a / t=0.2, D / t=17$ and $n=0.0446$. To be noted, the effects of SIF associated to the dimensions of the crack (as well as for different crack lengths) have also been considered. Figure 7a highlights the activity of the SIF. It increases with an increase in the crack length, and more particularly, the effects of crack length are observed more pronounced for short cracks. 
These results confirm that the deepest points (linked to crack front) in a pipe structure are the most dangerous locations in the cylinders with slender axial surface defects, conforming to $a / t<1$ (crack depth/cylinder thickness).

Besides, Fig. 7b proves the ascendant trend of the SIF values with respect to the crack size occurred in the pipe, crack dimensions as well as the record of $a / t$ for the external crack surface in the steel cylinder.

\section{Conclusions}

Based on the results of this paper, the following conclusions can be drawn:

- The analytical results presented in Figs. $7 \mathrm{a}$ and $7 \mathrm{~b}$ are found to be in good agreement with numerical results.

- A pipe with an axial external crack surface can be very well modelled using FE, as a three-dimensional finite element method, starting from a determined assumption. The FE modelling considers not only the cylindrical body but also the neck and transition areas of the cylinders, so the modelling process yields accurate results.

- The cylinder dimensions and crack size range are based directly on practically-applied steel gas cylinders, so the results are of greater practical value and can be applied to steel gas cylinders. The present results agree well with the results found in the literature.

- The Stress Intensity Factors associated with the axial crack surface are determined for different sizes. It has also been revealed that it is possible to describe the Stress Intensity Factor along the crack front of cylinders with axial external cracks by a six-order polynomial of the point of location. In short terms, this approach can be verified very well involving a second-order polynomial relative to the crack length $2 c / t$, and a third-order polynomial relative to the crack depth $a / t$.

\section{Acknowledgements}

This work was carried out with the assistance of the TSB as a part of the KTP scheme (KTP9299). We would also like to show our gratitude to Professor S. Hariri for his valuable insight.

\section{References}

1. Anderson P., 1999, Procedure for Safety Assessment of Component with Cracks, Handbook Swedish Nuclear Power Institute, Publication 99:49, SAQ/FOU Report 96/8. 3rd revised Ed.

2. Anderson T.L., 1884, Fracture Mechanics Fundamentals and Application, Chapter 12, Boca Raton, FL: CRC Press

3. ASME Boiler and Pressure Vessel Code, Section XI, 1981, Rules for in-service inspection of nuclear power plant components, American Soc. Mechanical Engineers, USA

4. CASTEM, 2014, http://www-cast3m.cea.fr/

5. Corigliano A., Maier G., Mariani S., 1999, Analysis of ductile fracture in damaged pipelines by a geometric parameter method, Engineering Structures, 21, 924-936

6. Cravero S., Ruggieri C., 2005, Correlation of fracture behavior in high pressure pipelines with axial flaws using constraint designed test specimens. Part I: Plane-strain analyses, Engineering Fracture Mechanics, 72, 1344-1360

7. Darcis P.P., McCowan C.N., Windhoff H., McColskey J.D., Siewert T.A., 2008, Crack tip opening angle optical measurement methods in five pipeline steels, Engineering Fracture Mechanics, 75, 2453-2468 
8. Heliot J., Labbens R.C., Pellissier-Tanon A., 1997, Semi-elliptical cracks in a cylinder subjected to stress gradients, Fracture Mechanics, ASTM STP 677, 341-364

9. LeE O.S., Choi S.S., 1999, Effect of circular cavity on maximum equivalent stress and stress intensity factor at a crack in buried pipeline, KSME International Journal, 13, 350357

10. McGowan J.J., Raymund M., 1997, Stress intensity factor solutions for internal longitudinal semi-elliptical surface flaws in a cylinder under arbitrary loading, Fracture Mechanics, ASTM STP 67r, $365-380$

11. Moustabchir H., Pruncu C.I., Azari Z., Hariri S., Dmytrakh I., 2015, Fracture mechanics defect assessment diagram on pipe from steel P264GH with a notch, International Journal of Mechanics and Materials in Design, DOI 10.1007/s10999-015-9296-z

12. Murtagian G.R., Johnson D.H., Ernst H.A., 2005, Dynamic crack propagation in steel line pipes. Part I: Experimental investigation, Engineering Fracture Mechanics, 72, 2519-2534

13. Nordhagen H., Dumoulin S., Gruben G., 2014, Main properties governing the ductile fracture velocity in pipelines: a numerical study using an (artificial fluid)-structure interaction model, 20th European Conference on Fracture (ECF20), Procedia Materials Science, 3, 1650-1655

14. RAJU I.S., NEWman JR J.C., 1982, Stress intensity factors for internal and external surface cracks in cylindrical vessels, Journal of Press Vessel Technology, 104, 293-308

15. Ruggieri C., Dotta F., 2011, Numerical modeling of ductile crack extension in high pressure pipelines with longitudinal flaws, Engineering Structures, 33, 1423-1438

16. Scheider I., Nonn A., Völling A., Mondry A., Kalwa C., 2014, A damage mechanics based evaluation of dynamic fracture resistance in gas pipelines, 20th European Conference on Fracture (ECF20), Procedia Materials Science, 3, 1956-1964

17. Schoots K., Rivera-Tinoco R., Verbong G., Van der ZwaAn B., 2011, Historical variation in the capital costs of natural gas, carbon dioxide and hydrogen pipelines and implications for future infrastructure, International Journal of Greenhouse Gas Control, 5, 1614-1623

18. Shin C.S., CAi C.Q., 2004, Experimental and finite element analyses on stress intensity factors of an elliptical surface crack in a circular shaft under tension and bending, International Journal of Fracture, 129, 239-264

19. StaAt M., Duc K.V., 2007, Limit analysis of flaws in pressurized pipes and cylindrical vessels. Part I: Axial defects, Engineering Fracture Mechanics, 74, 431-450

20. Stonesifer R.B., Brust F.W., Leis B.N., 1992, Stress intensity factors for long axial outer surface cracks in large $R / t$ pipes, Fracture Mechanics: Twenty-Second Symposium, ASTM STP $1131, \mathbf{1 1}, 29-45$

21. Su B., Bhuyan Gouri S., Elastic fracture properties of all-steel gas cylinders with different axial crack types, International Journal of Pressure Vessels and Piping, 76, 23-33

22. Tada H., Paris P.C., Irwin G.R., 2000, Stress Analysis of Cracks Handbook, Third Edition, ASME Press

23. TAn J.P., Tu S.T., Wang G.Z., Xuan F.Z., 2015, Characterization and correlation of 3-D creep constraint between axially cracked pipelines and test specimens, Engineering Fracture Mechanics

24. You L.H., Ou H., Zheng Z.Y., 2007, Creep deformations and stresses in thick-walled cylindrical vessels of functionally graded materials subjected to internal pressure, Composite Structures, 78, $2285-2291$

25. ZAhoor A., 1991, Ductile Fracture Handbook, Research project 1757-69, Vol. 3, Electric Power Research Institute 